\title{
Identification of a specific peptide binding to colon cancer cells from a phage-displayed peptide library
}

Lidan Hou ${ }^{1,6}$, Danxi Zhu ${ }^{1,6}$, Yu Liang ${ }^{1}$, Xiaohui Tian ${ }^{2}$, Lei Li ${ }^{1}$, Ping Wang ${ }^{1}$, Liming Zhu ${ }^{1}$, Xiaoling Weng ${ }^{3}$, Yingying $\mathrm{Wang}^{4}$, Yue $\mathrm{Li}^{5}$, Tianqi $\mathrm{Wu}^{3}$, Jianhua Wang ${ }^{\star, 3}$ and Xiangjun Meng ${ }^{\star, 1}$

${ }^{1}$ Department of Gastroenterology, Shanghai Ninth People's Hospital, Shanghai Jiao Tong University School of Medicine, Shanghai 200011, China; ${ }^{2}$ Department of Gastroenterology, Shanghai General Hospital, Shanghai 200060, China; ${ }^{3}$ Cancer institute, Fudan University Shanghai Cancer Center, Fudan University, Shanghai 200032, China; ${ }^{4}$ Department of Biochemistry and Molecular and Cell Biology, Shanghai Jiao Tong University School of Medicine, Shanghai 200025, China and ${ }^{5}$ Pathology Center, Shanghai First People's Hospital, Shanghai Jiao Tong University School of Medicine, Shanghai 200082, China

Background: New molecular probes are essential for early colon cancer diagnosis. A phage-display screening was performed to select novel binding peptides for early colon cancer imaging detection.

Methods: A human colon cancer cell line (COLO320HSR) and a normal human intestinal epithelial cell line (NCM460) were used for subtractive screening with a phage peptide library. The positive peptides were identified, and their binding capacities were confirmed by confocal immunofluorescence both in human colon cancer cells and in biopsy specimens. The sequences were further analysed for homology and the existing mimotopes by the BLAST algorithm and the MimoDB database.

Results: A peptide termed as CBP-DWS, which was demonstrated to be capable of binding to a panel of human colon cancer cell lines and tissues, was identified; it had virtually no binding to normal human intestinal epithelial cell line NCM460 and normal surrounding colon tissues. Bioinformatics analyses suggest that CBP-DWS targets human Glypican-3, which may be involved in important cellular functions in multiple cancer types.

Conclusions: These studies suggest that the selected peptide CBP-DWS may be a candidate to serve as a novel probe for colon cancer imaging.

Colorectal cancer (CRC) is the third most prevalent cancer diagnosed among men and women and the second leading cause of death from cancer in United States (Choi et al, 2017; Siegel et al, 2017). The 5year survival rate of patients with early-stage CRC reaches $90 \%$, while metastatic or late-stage colon cancer is only $10 \%$ (Garborg, 2015). In clinical practice, it is generally acknowledged that early diagnosis can be expected to enable implementation of personalised therapy that would help improve long-term outcomes. However, current screening modalities, such as the faecal occult blood test, faecal immunochemical test and colonoscopy, may be performed suboptimally, which can lead to the failure to detect neoplastic lesions and the performance of unnecessary endoscopic evaluations. Furthermore, a dysplastic lesion may be difficult to distinguish from epithelial regeneration associated with inflammation. Moreover, colonoscopy poses the risk of adverse events, such as perforation and post-polypectomy bleeding (Shahidi and Cheung, 2016). Consequently, it is a research priority to detect early CRC and develop sensitive diagnostic techniques.

\footnotetext{
*Correspondence: Professor J Wang; E-mail: jianhuaw2007@qq.com or Professor X Meng; E-mail: meng_xiangjun@yahoo.com

${ }^{6}$ These authors contributed equally to this work.
}

Received 13 June 2017; revised 8 September 2017; accepted 14 September 2017; published online 24 October 2017

(C) 2018 Cancer Research UK. All rights reserved 0007-0920/18 
Differences in the cell surface profile between cancer cells and their normal counterparts could help to identify molecular targets for tumour diagnosis and therapy. In the past decade, antibody engineering, especially highly specific, high-affinity monoclonal antibodies, have provided many advances in tumour diagnosis and targeted therapeutics (Kennedy et al, 2017). However, monoclonal antibodies are of limited efficacy especially against solid malignancies due in part to the size of the molecules, immunogenicity and poor pharmacokinetic properties (Krumpe and Mori, 2006). Comparing with antibodies, small peptides are typically nonimmunogenic, and their smaller size demonstrates much better tumour penetration while preventing non-specific uptake by the reticuloendothelial system. Notably, peptides show antibody-like affinities and selectivity for their receptors (Hamzeh-Mivehroud et al, 2013).

Phage display is a powerful technique that allows the presentation of multiple different peptides or proteins on the surface of phage particles, providing a means to select specific peptides or proteins that bind cancer cells even in the absence of the knowledge of the receptor (Bazan et al, 2012; Gray and Brown, 2014). A phage-display peptide library is comprised of a heterogeneous mixture of billions of phage clones, each carrying a different foreign DNA fragment and therefore displaying a different peptide on its surface. Screening of phage displayed peptide libraries using whole cells or intact tissues, often called biopanning, has proven to be successful for isolating peptides that show high cell and tissue specificities. To date, numerous studies have been performed to screen peptide-specific binding to cancer cells using phage display (Mandelin et al, 2015; Ma et al, 2016; Mann et al, 2016). Thus far, several peptides targeting colon cancer cells have also been reported, but there are some concerns with respect to specificity and sensitivity (Zhang et al, 2007; Liu et al, 2016). Herein, we aimed to find peptides with higher specificity for CRC imaging.

In our study, a peptide that specifically binds to human colon cancer cell line COLO320HSR but not to a normal human intestinal epithelial cell line NCM460 was selected by using in vitro phage-displayed peptide libraries. The results suggest that a peptide, CBP-DWS, can bind to colon cancer cells specifically and serve as a potential candidate of detection for colon cancer.

\section{MATERIALS AND METHODS}

Cell lines. The human colon cancer cell lines COLO320HSR, HCT116, SW480, HT29, LoVo were purchased from the American Type Culture Collection. A normal human intestinal epithelial cell line NCM460 was obtained from the Chinese Academy of Sciences, Shanghai Branch. COLO320HSR cells were grown in RPMI 1640 supplemented with $15 \%(\mathrm{v} / \mathrm{v})$ foetal bovine serum Gibco (Grand Island, NY, USA) and $0.015 \mathrm{mg} \mathrm{ml}^{-1} 5$-bromo-2'-deoxyuridine at $37^{\circ} \mathrm{C}$ in an atmosphere containing $5 \% \mathrm{CO}_{2}$. HT29 and SW480 were grown in DMEM (HyClone, Logan, UT, USA) supplemented with $10 \%(\mathrm{v} / \mathrm{v})$ foetal bovine serum. HCT116 were grown in McCoy's 5A (Gibco) with 10\% (v/v) foetal bovine serum. LoVo, NCM460 cells were grown in RPMI 1640 (Gibco) supplemented with $10 \%(\mathrm{v} / \mathrm{v})$ foetal bovine serum.

Whole-cell panning. A Ph.D.-12 phage-display peptide library kit was purchased from New England Biolabs (Ipswich, MA, USA). The library displayed 12 random peptides ligated at the $\mathrm{N}$-terminus of the minor coat protein (pIII) of M13 phage. The titre of library is $2 \times 10^{13}$ p.f.u. per $\mathrm{ml}$, and the complexity is $2.7 \times 10^{9}$ individual clones. The Escherichia coli host strain XL1 Blue (a robust $\mathrm{F}+$ strain with a rapid growth rate) was used for M13 phage propagation.
Screening procedures were performed according to the manufacturer's protocol, with some modifications. First, COLO320HSR cells were grown to nearly $80 \%$ confluence and collected into an Eppendorf tube. After washing with phosphatebuffered saline (PBS) three times, cells $\left(\sim 10^{7}\right.$ cells) were fixed in $4 \%$ paraformaldehyde $30 \mathrm{~min}$ and then blocked with $5 \%$ bovine serum albumin (BSA) to reduce non-specific hydrophobic binding. Subsequently, $1 \mathrm{ml}$ of phage-display peptide library that initially contained $2 \times 10^{12}$ p.f.u. per $100 \mu \mathrm{l}$ was added to the tube. The cells were incubated at room temperature with gentle shaking for $1 \mathrm{~h}$, and then centrifuged at 8000 r.p.m. for $3 \mathrm{~min}$. Then, the unbound phages were wiped off with $1 \mathrm{ml} \mathrm{1 \%} \mathrm{PBST} \mathrm{consisted} \mathrm{of} 1 \%$ Tween20 for four times. XL1 Blue (mid-log phage) of $0.5 \mathrm{ml}$ was added and incubated at $37^{\circ} \mathrm{C}$ for $1 \mathrm{~h}$. Subsequently, phage was titrated by a plaque-forming assay on agar plates containing tetracycline and amplified for the amplification of selected phage clones to be used in the next round of panning, according to the manufacturer's instructions. Four rounds of reiterative biopanning were performed. Finally, the selected phages were applied to normal human intestinal epithelial cell line NCM460 in the same way, for subtractive screening.

Binding affinity of selected phage clones. COLO320HSR cells were collected and fixed according to the methods described above. Each phage clones of $100 \mu \mathrm{l}$ to be tested were added to $1 \mathrm{ml} \mathrm{XL1}$ Blue (mid-log phage), respectively, and incubated at $37^{\circ} \mathrm{C}$ for $1 \mathrm{~h}$. The mixture was centrifuged at 10000 r.p.m. for $5 \mathrm{~min}$ and then added the supernatant to COLO320HSR cells, allowed to bind for $1 \mathrm{~h}$, and then unbound phage was removed with $0.5 \%$ PBST with $0.5 \%$ Tween at 8000 r.p.m., for $3 \mathrm{~min}$ four repetitions. The unselected original phages were used as the control group. Positive phage clones were determined by an average enrichment rate of 10 times that of the negative control group. The average enrichment rate was calculated as output number/input number.

DNA sequencing of positive phage clones. Single-stranded DNA from the positive phages was prepared according to a standard protocol, using iodide buffer (10 mM Tris- $\mathrm{HCl}, 1 \mathrm{~mm}$ EDTA and $4 \mathrm{M}$ NaI, pH 8.0 (Riedel-de Haen)), followed by ethanol precipitation. The pellet was resuspended in $30 \mu \mathrm{l}$ of Tris-EDTA buffer (TE buffer $1 \times: 0.1 \mathrm{M}$ Tris-base $\mathrm{pH} 8.0$ and $10 \mathrm{~mm}$ EDTA) and the product was quantified using Nanodrop1000 (Thermo Scientific, Waltham, MA, USA) and sent to Shanghai Sangon Corp. (Shanghai, China) for sequencing. The BioEdit Sequence Alignment Editor software and the ProtParam programs (Ibis Biosciences, Carlsbad, CA, USA) were used to analyse DNA sequences of the positive phage clones, taking into account that the displayed peptides are expressed at the N-terminus of pIII, followed by a short spacer (Gly-Gly-Gly-Ser, GGGS) and then the wild-type pIII sequence. The primer used for sequencing was 5'-CCGATACAATTAAAGGCTCC-3' (96gIII sequencing primer, New England Biolabs).

Peptide synthesis and labelling. The peptide CBP-DWS (DWSSWVYRDPQT) and a scrambled peptide CBP-SCR (DLPSRTSALASG) were synthesised on solid phase using a rapid and practical Fmoc method, and they were purified by reverse phase high-performance liquid chromatography (Agilent Technologies, Santa Clara, CA, USA). High purity (>98\%) was obtained after purification. The synthetic peptide was further characterised with respect to its identity by mass spectrometry analysis. Peptide were synthesised and labelled with fluorescein isothiocyanate (FITC) at the N-terminus by Shanghai HD Biosciences Corporation (Shanghai, China).

Fluorescent peptide binding to cancer cells. COLO320HSR, NCM460, HCT116, HT29, SW480, LoVo cells were grown on coverslips overnight and fixed with $4 \%$ paraformaldehyde at room temperature for $30 \mathrm{~min}$. Samples were blocked in 3\% BSA (w/v) in 
PBS for $30 \mathrm{~min}$ at room temperature. Cells were then incubated with $10 \mu \mathrm{M}$ FITC-labelled peptides for $15 \mathrm{~min}$ at room temperature and washed with PBS for three times. This was followed by nuclear staining with 4,6-diamidino-2-phenylindole (DAPI). A laser scanning confocal microscope was used to visualise the slides (Nikon, Tokyo, Japan). The cumulative values of the integrated optical density (IOD) of green fluorescence (FITC) in each photo were analysed using the Image-Pro Plus 6.0 software (Media Cybernetics, Inc., Rockville, MD, USA). The average value of the IOD was expressed as the mean \pm s.d.

FITC-CBP-DWS and scrambled peptide FITC-CBP-SCR were serially diluted in PBS at increasing concentrations $(0-320 \mu \mathrm{M})$ and incubated with human colon cancer cells (HCT116 and LoVo cells) and normal colonic epithelial cell line NCM460 at $37^{\circ} \mathrm{C}$ for $1 \mathrm{~h}$. Subsequently, these cells were resuspended in $200 \mathrm{ml}$ PBS and transferred to a 96-well black clear bottom costar plate. The fluorescence intensity was measured at $525 \mathrm{~nm}$ using excitation at $488 \mathrm{~nm}$ in a multi-well plate reader (BioTek synergy4, BioTek Instruments Inc., Winooski, VT, USA).
For the competitive inhibition assay, HCT116 and LoVo cells were first incubated with several concentrations (10, 100 and $200 \mu \mathrm{M})$ of unlabelled free peptide CBP-DWS in PBS for $1 \mathrm{~h}$. Then, the cells were washed and incubated with FITC-labelled CBPDWS for additional $15 \mathrm{~min}$ as described above. The slips were observed using a fluorescence microscope (Olympus, Tokyo, Japan). Each experiment was carried out in duplicate and repeated twice.

Fluorescent peptide-binding capacity ex vivo. Six pairs of fresh colon cancer tissues and adjacent normal tissues were collected from Tong Ren Hospital Shanghai, Jiao Tong University School of Medicine. Only patients who had not received chemotherapy or radiotherapy before surgery were selected. Tissues were obtained immediately after surgery, washed twice with chilled PBS, immediately embedded in optimal cutting temperature medium, and then cut into $7 \mu \mathrm{m}$-thick frozen sections.

Tissue slides were blocked with 3\% BSA for $30 \mathrm{~min}$. Next, the tissues were incubated with $200 \mu \mathrm{l}$ of FITC-CBP-DWS $(20 \mu \mathrm{M})$ for $15 \mathrm{~min}$ at room temperature in a chamber kept away from light.

A
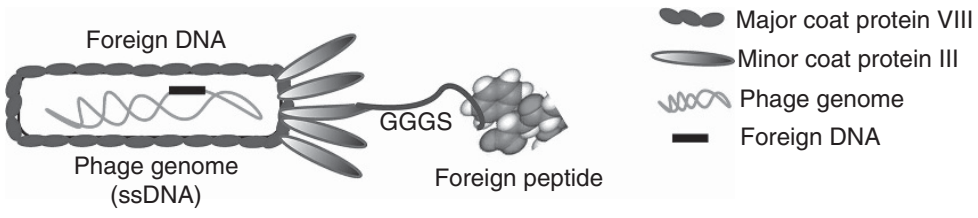

(ssDNA)

- Foreign DNA

B

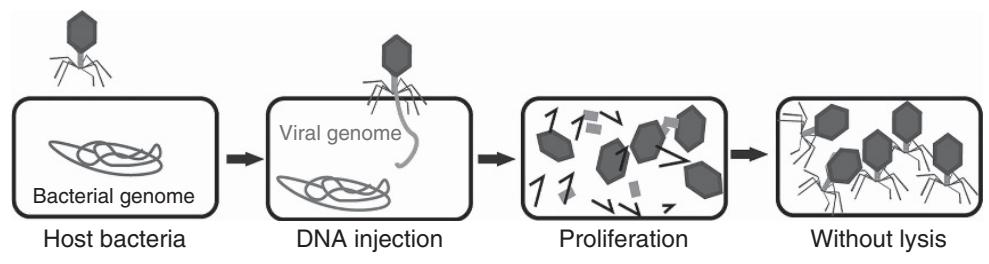

C

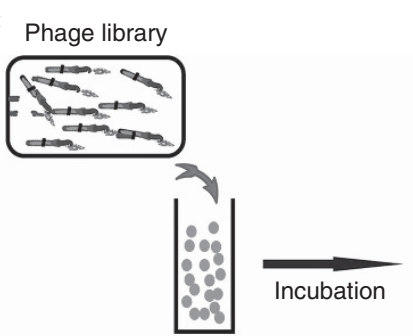

COLO32OHSR cells

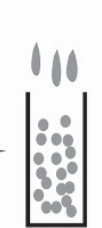

Discard unbound phage

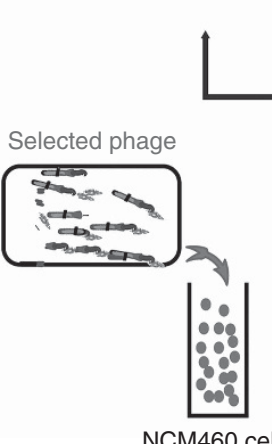

Wash
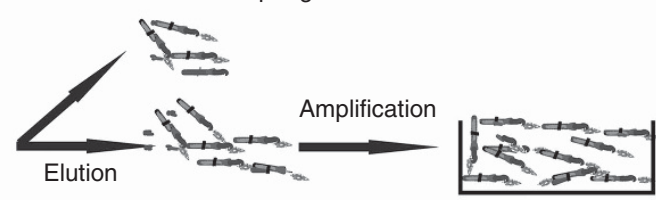

Bound phage

XL1-Blue with bound phage

Repeat 4 rounds

NCM460 cells
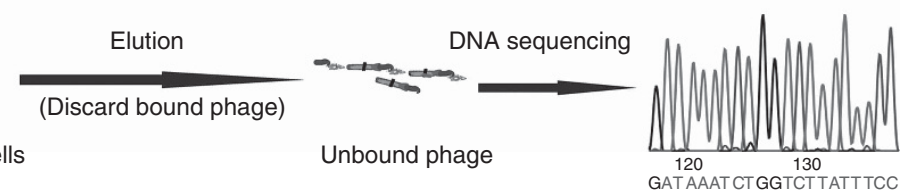

Figure 1. Selection of specific peptides to COLO320HSR cells by phage display. (A) Schematic representation of M13 filamentous phage. Random foreign DNA fragments are inserted into the phage genomes. The random peptide is shown fused to the amino terminus of the plll coat protein by a short spacer (Gly-Gly-Gly-Ser, GGGS). (B) Life cycle of non-lytic filamentous phages in host bacteria. The binding of plll to the bacteria cell membrane leads to injection of phage ssDNA into the bacteria. New phage DNA and proteins are synthesised and then phages are assembled. (C) Workflow of phage-display-based screening in vitro. After four rounds of screening on human colon cancer cells COLO320HSR plus a subtractive screening on normal human intestinal epithelial cells NCM460, cancer cell-bound phages were isolated and sequenced. A full colour version of this figure is available at the British Journal of Cancer journal online. 

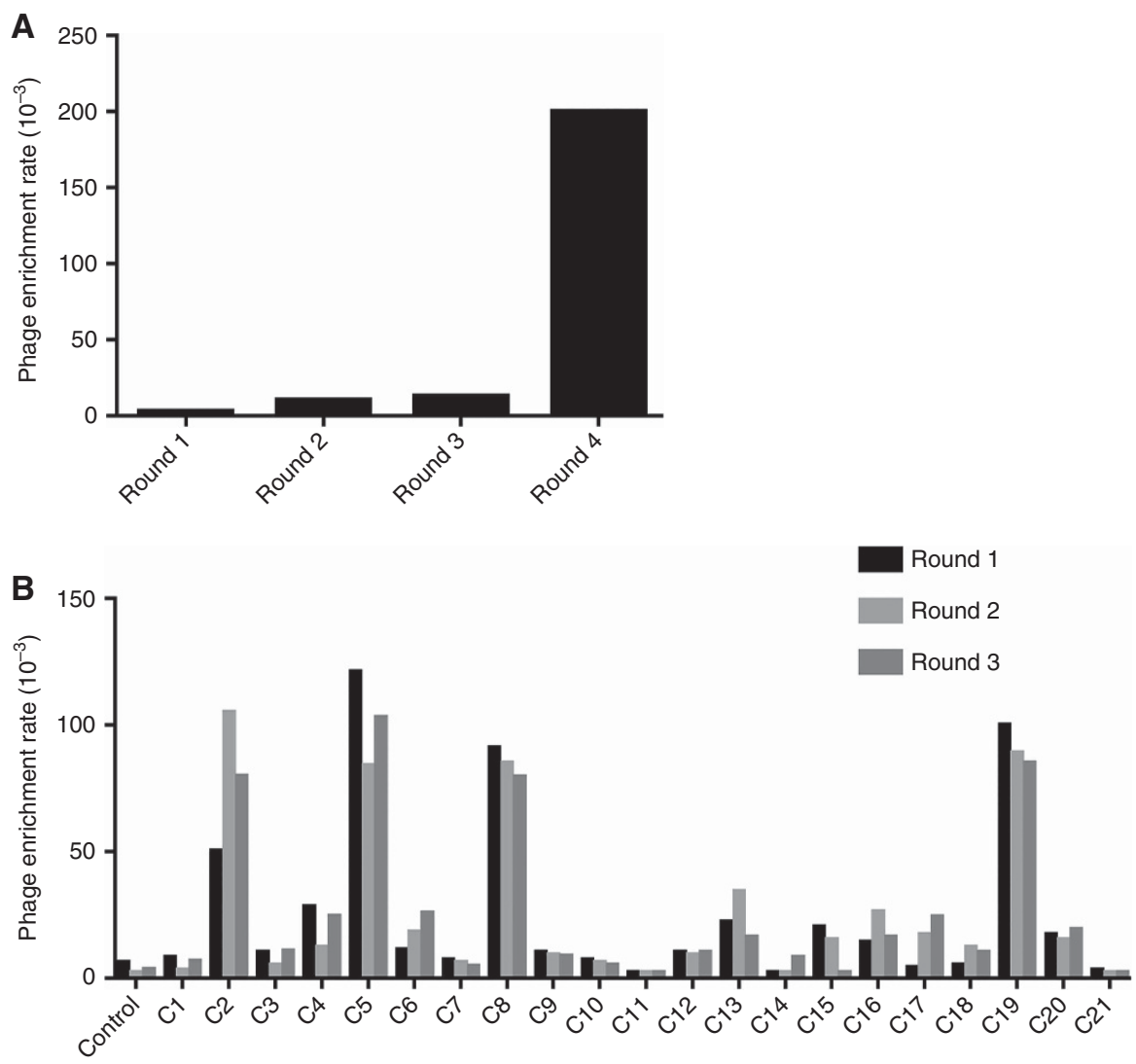

C

DWSSWVYRDPQTGGGS

CTATTCTCACTCTGATTGGAGTTCGTGGGTGTATAGGGATCCGCAGACTGGTGGAGGTTCGGCCGAAACTGTTG

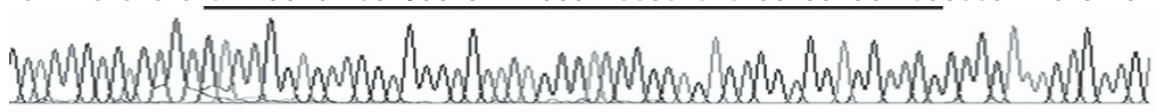

LPKTVSSDMSLNGGGS

CTATTCTCACTCTCTTCCTAAGACTGTTTCGTCTGATATGTCTTTGAATGGTGGAGG TTCGGCCGAAACTGTTG

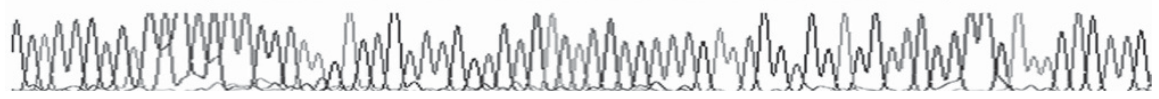

Figure 2. Specific binding of the positive phage clones to COLO320HSR cells. (A) The titres of recovered phages from each round were evaluated by blue plaque-forming assay on an agar plate containing tetracycline. The phage enrichment rate was calculated as output number/input number. (B) After four rounds of biopanning, 21 plaques were randomly selected and analysed by repeated screening on COLO320HSR cells. Four phage clones (C2, C5, C8, C19) appeared to have higher specific binding ability than other clones and were considered as positive phage clones. The unselected original phages were used as negative controls. (C) Amino acid sequence analysis of phage positive clones. The DNA sequences of positive phage clones were sequenced and translated into peptide sequences according to the triple-code theory. A full colour version of this figure is available at the British Journal of Cancer journal online.

Afterward, the slides were rinsed for three times with $0.1 \%(\mathrm{v} / \mathrm{v})$ Tween-20 in TBS five times, and mounted with DAPI. A FITClabelled scrambled peptide was used as a negative control. The slips were examined using a fluorescence microscope (Olympus). Each experiment was carried out in duplicate and repeated twice. The same tissue slides were diagnosed through haematoxylin and eosin staining. The images were analysed by Image-Pro Plus 6.0 software as described above.

Peptide analysis and verification. Gene sequences were analysed by the BLAST algorithm for homology to proteins with known or putative cancer correlations. The query was performed using the BLAST search program (BLASTP 2.6.1+) against the Homo sapiens non-redundant protein database using Blastp (http://ncbi.nlm.nih.gov/blast/). The identified sequences were further scanned using the SAROTUP webserver software (http://immunet.cn/sarotup/) and the MimoDB database for already existing mimotopes.

To determine the cell surface expression pattern of Glypican-3 receptor, HCT116 and LoVo cells were fixed with 4\% paraformaldehyde for $30 \mathrm{~min}$. After blocking with 5\% BSA for $1 \mathrm{~h}$, fixed cells were incubated with sheep anti-human Glypican-3 (1:800, R\&D Systems, Minneapolis, MN, USA) at $4{ }^{\circ} \mathrm{C}$ overnight. They were subsequently stained with NorthernLights 557 conjugated antisheep IgG secondary antibody (1:200, R\&D Systems) for $1 \mathrm{~h}$ at room temperature. After washing three times with PBS, cells were mounted with DAPI and observed using a fluorescence microscope (Olympus).

Statistical analysis. GraphPad Prism 7.0 (GraphPad Software, Inc., San Diego, CA, USA) was used for statistical analysis of the data. The significance of the difference from the respective controls 


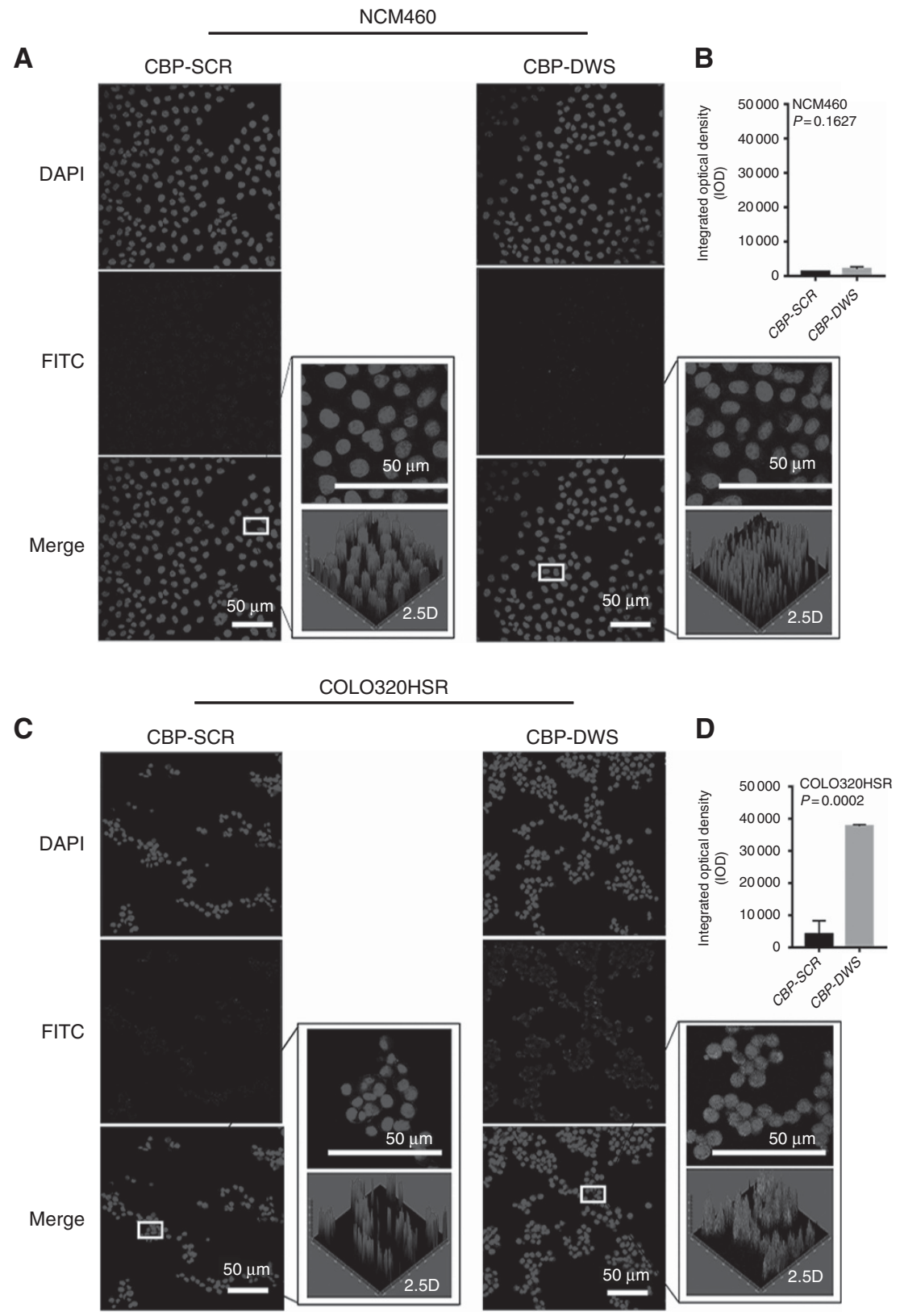

Figure 3. CBP- DWS shows selective homing to COLO32OHSR cells. Fixed human colon cancer COLO32OHSR cells and normal human intestinal epithelial NCM460 cells were stained with $10 \mu \mathrm{ml}^{-1}$ FITC-CBP for $30 \mathrm{~min}$. Strong fluorescent signals were observed on COLO32OHSR cells while quite few was detected on NCM460 cells (A, C). Images are also presented in a 2.5-dimension format. The IOD was analysed by Image-Pro Plus 6.0 (B, D). Green colour is from FITC for CBP-DWS. Blue colour is from DAPI for visualising cell nuclei. Scale bar $50 \mu \mathrm{m}$. A full colour version of this figure is available at the British Journal of Cancer journal online.

for each experimental test condition was assessed using the Student's $t$ test for each paired experiment.

\section{RESULTS}

Selection of the COLO320HSR specifically binding phage clones. The phage-display system used in this study is based on a simple non-lytic filamentous M13 phage vector. The filamentous phage is a flexible rod composed of capsid protein encasing a circular single strand of DNA. Random foreign DNA fragments are inserted into the phage genomes. M13 phages are modified for pentavalent display of peptides as N-terminal fusions to the minor coat protein pIII by a short linker GGGS (Figure 1A). Non-lytic filamentous phages, which assemble in and secrete from their bacterial hosts without bacterial cell lysis, are commonly used for library construction (Figure 1B). Four selection rounds were performed on the COLO320HSR cell line to allow for enrichment of tumour cell binding or internalising phages. Subsequently, a negative selection with the normal human intestinal epithelial cell line NCM460 was done to subtract phages that bound to nontumour cells (Figure 1C). The output/input ratio of phages (phage enrichment rate) recovered after each round of the panning were used to determine the phage recovery efficiency. As more and more unbound phage clones were discarded, the bound phages were rescued and titrated (Supplementary Table 1) and phage enrichment rate gradually increased. The number of phages recovered from COLO320HSR cells increased 60 -fold $\left(3 \times 10^{3}\right.$ vs $\left.5 \times 10^{-5}\right)$ after the final selection round (Figure 2A). Eventually, 21 phages clones were selected for further testing. 
A

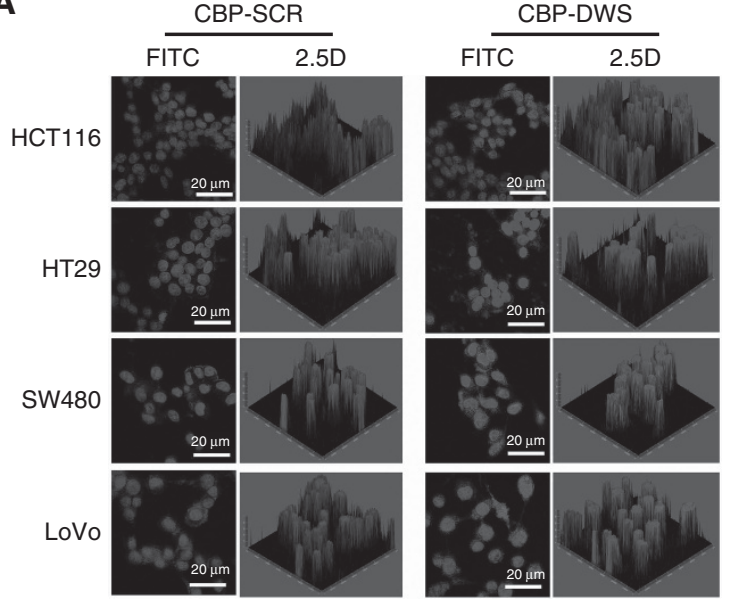

E

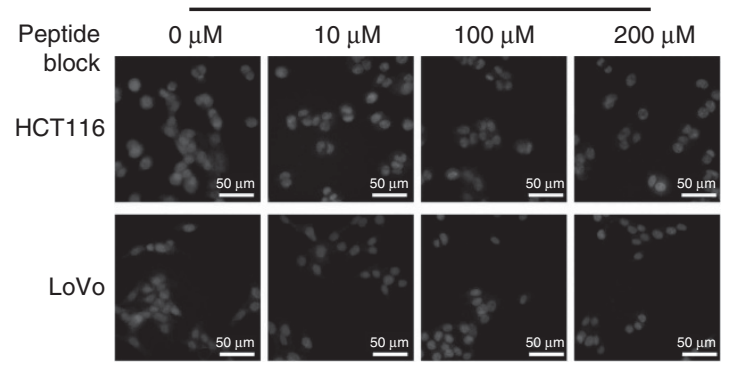

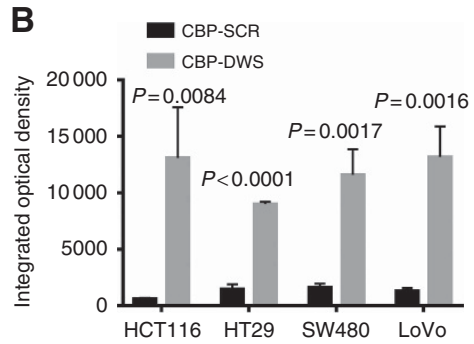

C

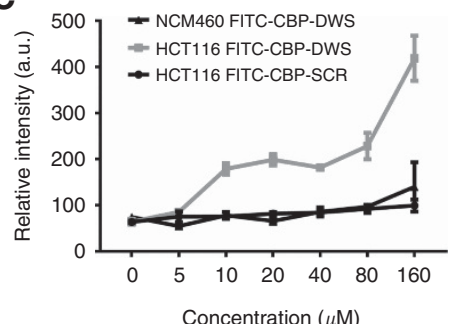

D

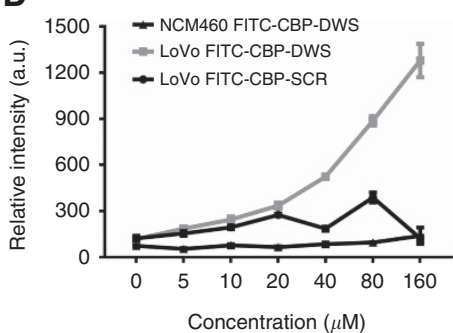

Figure 4. Fluorescent imaging of CBP-DWS binding to a panel of human colon cancer cells. (A) The FITC-labelled peptide CBP-DWS bound to a panel of human colon cancer cells HCT116, HT29, SW480, LoVo, whereas the FITC-labelled control peptide CBP-SCR bound with low affinity to them. Images are also presented in a 2.5-dimension format. Scale bar $20 \mu \mathrm{m}$. (B) The IOD of CBP-DWS was significantly higher than that of CBPSCR. The binding efficiency of the fluorescent peptide to HCT116 (C) and LoVo cells (D) were determined. (E) Unlabelled peptide CBP-DWS were used for competitive inhibition of FITC-CBP-DWS. The fluorescence intensity of FITC-CBP-DWS decreased on both HCT116 and LoVo cells. Scale bar $50 \mu \mathrm{m}$. A full colour version of this figure is available at the British Journal of Cancer journal online.

Affinity confirmation of selected phage clones. To further confirm the affinity of the 21 selected phage colons to COLO320HSR, the binding capacity of these phage clones to COLO320HSR cells was repeatedly verified on COLO320HSR cells in liquid phase. The average enrichment rate was calculated as output/input phage number. Ultimately, only four clones (C2, C5, $\mathrm{C} 18$, C19) were showed to be a significantly higher phage recovery rate compared to other clones ( $>10$ times higher), indicating that they had a strong capacity to bind to COLO320HSR cells (Figure 2B). Therefore, the four phage clones were considered as positive clones.

DNA sequencing of the positive phage clones. The ssDNAs of the positive phage clones were then sequenced and analysed. As shown in Figure 2C, the peptide sequences displayed on the positive phage clones were deduced from the DNA sequences corroding to triple-code theory. Because the location of the insertion of exogenous DNA into E. coli is consistent, the DNA sequence of the start and end of each clone is the same. One sequence (DWSSWVYRDPQT) was eventually found to be enriched in three of the four positive phage clones. The peptide was termed as CBP-DWS. To provide a control peptide, we synthesised a scramble peptide, DLPSRTSALASG, which we named CBP-SCR.

Validation of peptide binding to human cancer cells in vitro. To confirm the binding ability of the selected peptides to COLO320HSR cells, the CBP-DWS peptide was synthesised and conjugated with green florescent FITC at the N-terminus of the peptide. Cells were stained with FITC-CBP-DWS or FITC-CBP-SCR $(10 \mu \mathrm{M})$ in vitro.
FITC-CBP-DWS showed abundant binding to COLO320HSR cells, whereas it showed almost no binding to the control cells NCM460 (Figure $3 \mathrm{~A}$ and $\mathrm{C}$ ). Images were also presented in a 2.5-dimension format. The intensity of green fluorescence signal was calculated, and the values were shown in Figure $3 \mathrm{~B}$ and D. The fluorescence intensity of CBP-DWS when applied to COLO320HSR and NCM460 cells was significantly different.

Similar trends in the binding capacity were observed on other four human colon cancer cells HCT116, HT29, SW480, LoVo. A strong fluorescent signal was observed in each of these cell lines. In contrast, the scrambled peptide FITC-CBP-SCR exhibited almost no positive fluorescence with any of the cell lines (Figure 4A and B). FACS assay revealed that CBP-DWS was also capable of markedly binding to HCT116 and LoVo, as compared with their respective controls. The binding was found to be stronger than binding to NCM460 (Supplementary Figure 1). We further test the binding efficiency of FITC-CBP-DWS to cells. The results showed that, compared to normal cells, the intensity of the fluorescence increased with increasing concentration of FITC-CBP-DWS in HCT116 and LoVo cells. However, the CBP-SCR peptide did not display the same tendency (Figure $4 \mathrm{C}$ and $\mathrm{D}$ ). Immunofluorescence staining of different concentrations of CBP-DWS are shown in Supplementary Figure 2. In addition, the inhibitory effects of the free unlabelled peptide on CBP-DWS-binding ability were examined. After pre-incubation with increasing concentrations of the unlabelled peptide, HCT116 and LoVo cells were incubated with $10 \mu \mathrm{M}$ FITC-CBP-DWS. The results, presented in Figure 4E, show a decreased binding with increasing concentrations of unlabelled peptide from 0 to $200 \mu \mathrm{m}$ in both cell lines. Taken 
A
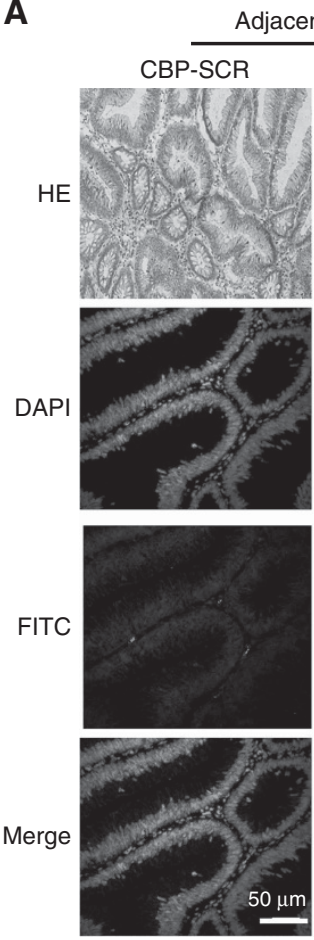

Adjacent normal

CBP-DWS
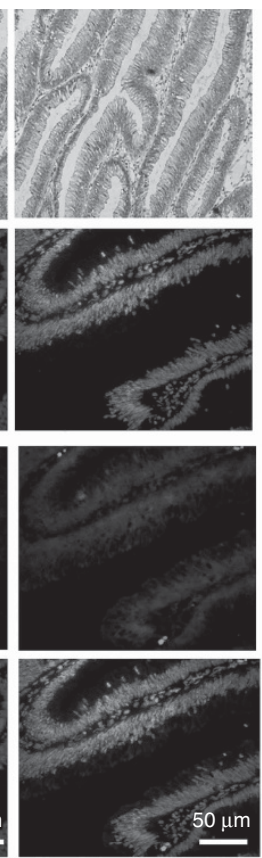
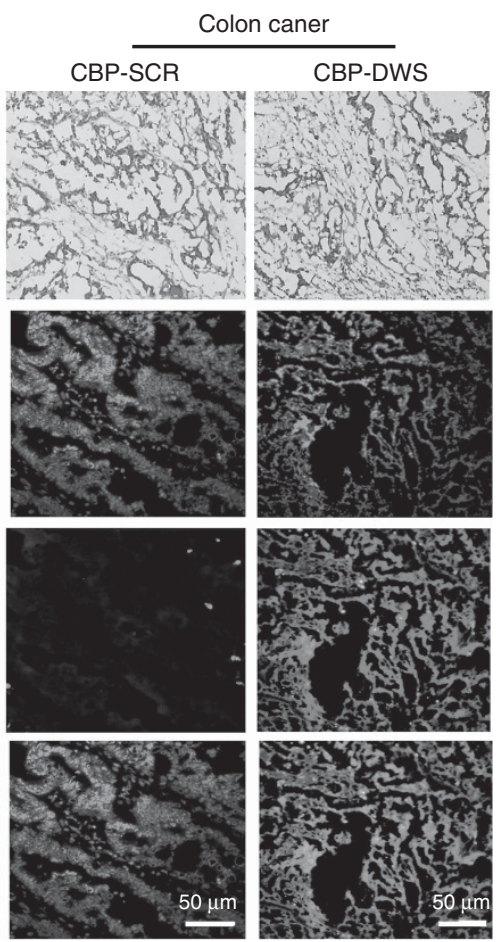

B

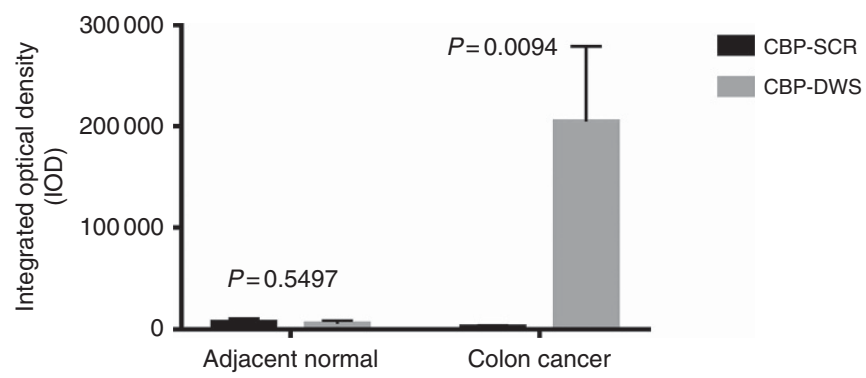

Figure 5. Ex vivo peptide binding to human colon specimens. (A) Frozen serial sections of matched human colon cancer and adjacent normal tissues were stained with $20 \mu \mathrm{m}$ FITC-CBP-SCR or FITC-CBP-DWS at room temperature for $30 \mathrm{~min}(n=6)$. Green colour is from FITC, and blue colour from DAPI for nuclear visualisation. Tissue sections were also stained with H\&E and imaged with a digital light microscope. Magnification, $\times 200$. Scale bars: $50 \mu \mathrm{m}$. (B) Fluorescence signal intensity analysis reveals CBP-DWS had a significantly higher affinity for colon cancer tissues than CBP-SCR $(P=0.0094)$. A full colour version of this figure is available at the British Journal of Cancer journal online.

together, these findings demonstrated that the identified peptide has specific binding to human colon cancer cells.

Validation of peptide binding ex vivo. On the basis of the binding of CBP-DWS to a panel of human colon cancer cell lines, we next assayed the effect of CBP-DWS-binding human colon tumour tissues. Immunofluorescence analysis was performed on frozen human colon adenocarcinoma and adjacent normal colon tissues. We observed that FITC-CBP-DWS showed preferential binding to human colon cancer tissues, whereas no apparent staining signals were observed in adjacent normal tumour sections (Figure 5A). The selective binding of FITC-CBP-DWS to colon cancer tissues was further confirmed by using the scrambled peptide CBP-SCR, which showed no binding to colon tumour as shown in Figure 5B $(P<0.01)$.

Peptide analysis and homology. The peptide CBP-DWS was analysed using protein-protein blast BLASTP 2.6.0 + for homology. Top matching proteins were evaluated for per cent homology, E-value (Supplementary Table 2). Our results revealed that no peptide sequence was homologous to CBP-DWS. The molecular target of the CBP-DWS is unknown and the sequence does not show full homology to ligands for known receptors. CBP-DWS was further scanned using the SAROTUP webserver software and the MimoDB database to screen for published mimotopes of other groups with various target peptide. Glypican-3 was shown to be a potential receptor for CBP (Supplementary Table 3).

We first checked the expression pattern of the glypican-3 receptor in HCT116 and LoVo cells by using human glypican-3 antibody staining. Figure 6 shows that glypican-3 is abundantly expressed on HCT116 and LoVo cells. We then tested the glypican-3 receptor specificity of CBP-DWS peptide. Our data indicate that pre-incubation with CBP-DWS peptide abrogated the binding of glypican-3 antibody to HCT116 and LoVo cells.

\section{DISCUSSION}

Whole-cell panning was performed on human colon cancer cells COLO320HSR. The phage-display system is based on a simple M13 phage vector modified for pentavalent display of peptides as $\mathrm{N}$-terminal fusions to the minor coat protein pIII by a short spacer GGGS (Figure 1A). The binding effect depends on the distribution density of the various molecules expressed on the surface of the cell, the type of ligand, the exposure degree of 


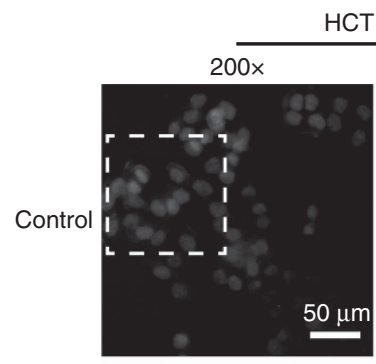

HCT116
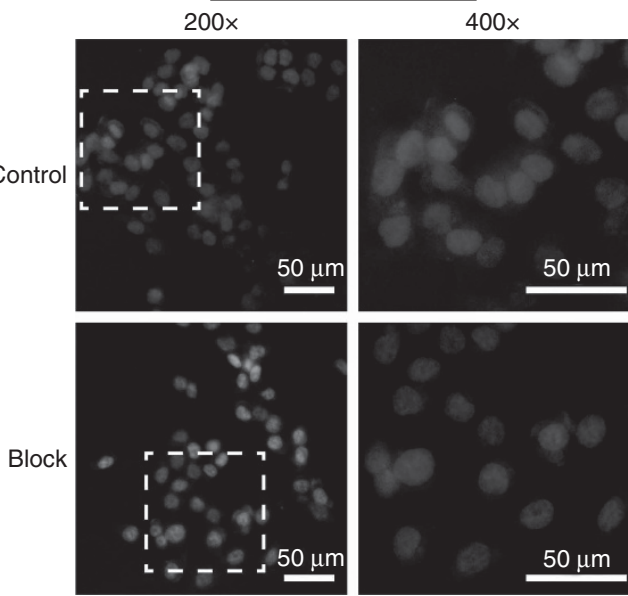

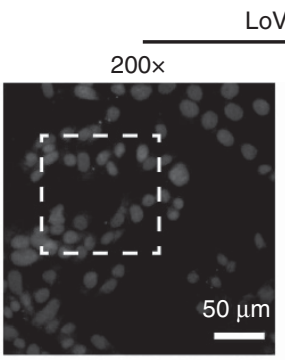

oVo
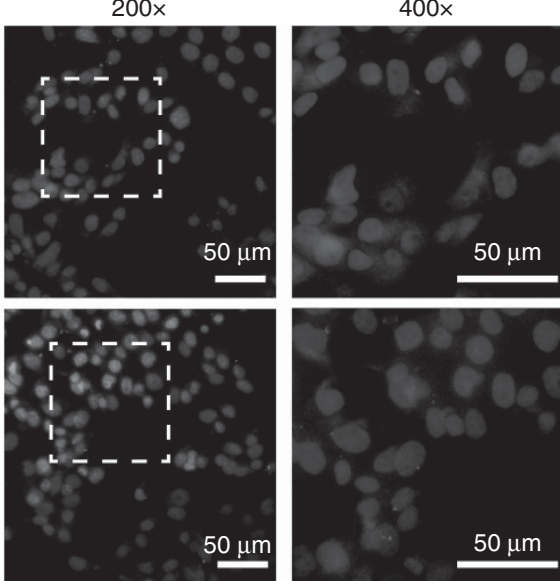

Figure 6. Immunofluorescence staining of glypican-3 without and with CBP-DWS blocking. Glypican-3 was detected in HCT116 and LoVo cell lines using sheep anti-human Glypican-3 at $4{ }^{\circ} \mathrm{C}$ overnight. And then cells were stained using NorthernLightsTM 557 conjugated anti-sheep secondary antibody (red) and counterstained with DAPI (blue). Bar, $50 \mu \mathrm{m}$. A full colour version of this figure is available at the British Journal of Cancer journal online.

the cell surface molecule, and its affinity to the phage peptides (Helms et al, 2009). A normal colonic epithelial cell line NCM460 was used as a substantive screen to exclude peptides that bind non-specifically to cancer cells. The phage enrichment rate was gradually increased with four rounds of screening, indicating that the phage clones with binding ability were effectively enriched (Figure 2A).

COLO320HSR cells are easily washed down and hard to immobilise on the ELISA plate due to its loosely adherent state, so we validated the specificity of phage peptides in the liquid phase (PBS). The washing procedure in the liquid phase environment is much more efficacious than that when cells are on a solid surface; therefore, the effect of non-specific binding could be better reduced. Because the dominant clones are always preferentially amplified and some minor clones are rapidly reduced in the amplified libraries due to the phylogenetic library bias (Matochko et al, 2014), the original library was used each time to minimise the effect of the amplified phage library on our results. The positive phage clones were found to have an average enrichment rate of 10 times higher than the negative control group. Twenty-one phage clones were randomly selected, but only four clones were stably flattened $>10$ times than the negative control group, demonstrating that they had higher specific binding ability to colon cancer COLO320HSR (Figure 2B). Subsequently DNA sequencing revealed that three of them have the same sequence DWSSWVYRDPQT (Figure 2C).

Like the phage-bound peptide, free peptide CBP-DWS again showed specificity and selectivity to COLO320HSR cells relative to the NCM460 cells as shown in Figure 3. We further observed the cell IOD of FITC-labelled CBP-DWS were 10-100-fold higher than FITC-labelled CBP-SCR (Figure 4A and B). Fluorescence intensity increased with an increase in concentration of FITC-CBP-DWS for both HCT116 and LoVo cells (Figure 4C and D). After blocking the cells with free unlabelled peptide, the fluorescence intensity was significantly reduced, indicating that CBP-DWS had a binding specific to these cancer cells (Figure 4E). Meanwhile, six pairs of human colon cancer tissue and adjacent normal tissues were tested for cell specificity ex vivo and CBP-DWS also showed specificity for the cancer tissues (Figure 5).

The results above suggest that CBP-DWS likely recognises a receptor that is selectively expressed on the surface of tumour cells. Identification of the receptors responsible for peptide binding may allow for the discovery of novel cell surface molecules, which may yield future targets for drug design. However, we did not find any sequence fully homologous to CBP-DWS by the BLAST algorithm, highlighting the novelty of the identified sequences.

Next, we compared the peptide to the already existing mimotopes. CBP-DWS were scanned using the SAROTUP webserver software and the MimoDB database to screen for published mimotopes of other groups with various target peptides (Huang et al, 2010; He et al, 2016). Glypican-3 were shown to be a potential receptor for CBPDWS (Zhu et al, 2016). Glypican-3, composed of a core protein and two heparansulfate chains, is a key member of the glypican family that is expressed on the cell surface by a glycosyl-phosphatidylinositol (GPI) anchor (Filmus et al, 2008). It was reported that Glypican-3 might have an important role in the genesis and development of several tumours, such as hepatocellular carcinomas, ovarian carcinoma and CRC (Filmus and Capurro, 2013; De Robertis et al, 2015; Suzuki et al, 2016). Therefore, it is highly reasonable to surmise that the binding targets of CBP-DWS on the surface of colon cancer cells are Glypican-3. Our data confirmed that human colon cancer cell HCT116 and LoVo overexpress Glypican-3 and that antiGlypican-3 antibody binding to colon cancer cells can be blocked by excess amount of CBP-DWS peptide. Future analysis underlying docking for Glypican-3 could be interesting to prove its role as a targeting receptor of CBP-DWS. While we do not exactly know the functional area of the cell-specific peptides CBP-DWS, it is interesting to note that it contains a $\beta$-strand, which may have an important role in the interaction between the peptide and the receptor (Robinson et al, 2015; Hsu et al, 2017). We are in the process of trying to identify the cellular receptor(s) for this peptide.

In conclusion, the linear 12-mer peptide CBP-DWS obtained by phage-display technology showed significant ability to bind to colon cancer cells and tissues. Because of its high and specific binding to colon cancer cells in vitro and ex vivo, CBP-DWS may be used as a malignant tumour-targeting tracer and as a targeted therapy for early treatment of colon cancer. Further studies of the mechanism of CBP-DWS binding are warranted, as are studies to verify detection of colon cancer in animal models.

\section{ACKNOWLEDGEMENTS}

This work was supported by the National Natural Funding of China (81272404, 81772806), National Key Program (973) for Basic Research of China (2011CB510106), the Program for Professor of Special Appointment (Eastern Scholar to JW) at Shanghai Institutions of Higher Learning. 


\section{CONFLICT OF INTEREST}

The authors declare no conflict of interest.

\section{REFERENCES}

Bazan J, Calkosinski I, Gamian A (2012) Phage display-a powerful technique for immunotherapy: 1. Introduction and potential of therapeutic applications. Hum Vacc Immunother 8(12): 1817-1828.

Choi Y, Sateia HF, Peairs KS, Stewart RW (2017) Screening for colorectal cancer. Semin Oncol 44(1): 34-44.

De Robertis M, Arigoni M, Loiacono L, Riccardo F, Calogero RA, Feodorova Y, Tashkova D, Belovejdov V, Sarafian V, Cavallo F, Signori E (2015) Novel insights into Notum and glypicans regulation in colorectal cancer. Oncotarget 6(38): 41237-41257.

Filmus J, Capurro M (2013) Glypican-3: a marker and a therapeutic target in hepatocellular carcinoma. FEBS J 280(10): 2471-2476.

Filmus J, Capurro M, Rast J (2008) Glypicans. Genome Biol 9(5): 224.

Garborg K (2015) Colorectal cancer screening. Surg Clin North Am 95(5): 979-989.

Gray BP, Brown KC (2014) Combinatorial peptide libraries: mining for cellbinding peptides. Chem Rev 114(2): 1020-1081.

Hamzeh-Mivehroud M, Alizadeh AA, Morris MB, Church WB, Dastmalchi S (2013) Phage display as a technology delivering on the promise of peptide drug discovery. Drug Discov Today 18(23-24): 1144-1157.

He B, Chai G, Duan Y, Yan Z, Qiu L, Zhang H, Liu Z, He Q, Han K, Ru B, Guo FB, Ding H, Lin H, Wang X, Rao N, Zhou P, Huang J (2016) BDB: biopanning data bank. Nucleic Acids Res 44(D1): D1127-D1132.

Helms BA, Reulen SW, Nijhuis S, de Graaf-Heuvelmans PT, Merkx M, Meijer EW (2009) High-affinity peptide-based collagen targeting using synthetic phage mimics: from phage display to dendrimer display. J Am Chem Soc 131(33): 11683-11685.

Hsu DS, Hwang WL, Yuh $\mathrm{CH}$, Chu $\mathrm{CH}$, Ho YH, Chen PB, Lin HS, Lin HK, Wu SP, Lin CY, Hsu WH, Lan HY, Wang HJ, Tai SK, Hung MC, Yang MH (2017) Lymphotoxin-beta interacts with methylated EGFR to mediate acquired resistance to cetuximab in head and neck cancer. Clin Cancer Res 23(15): 4388-4401.

Huang J, Ru B, Li S, Lin H, Guo FB (2010) SAROTUP: scanner and reporter of target-unrelated peptides. J Biomed Biotechnol 2010: 101932.

Kennedy PJ, Oliveira C, Granja PL, Sarmento B (2017) Antibodies and associates: partners in targeted drug delivery. Pharmacol Ther 177: 129-145.

Krumpe LR, Mori T (2006) The use of phage-displayed peptide libraries to develop tumor-targeting drugs. Int J Pept Res Ther 12(1): 79-91.
Liu Z, Gray BD, Barber C, Bernas M, Cai M, Furenlid LR, Rouse A, Patel C, Banerjee B, Liang R, Gmitro AF, Witte MH, Pak KY, Woolfenden JM (2016) Characterization of TCP-1 probes for molecular imaging of colon cancer. J Control Release 239: 223-230.

Ma Z, Qin H, Chen H, Yang H, Xu J, Yang S, Hu J, Xing D (2016) Phage display-derived oligopeptide-functionalized probes for in vivo specific photoacoustic imaging of osteosarcoma. Nanomedicine 13: 111-121.

Mandelin J, Cardo-Vila M, Driessen WH, Mathew P, Navone NM, Lin SH, Logothetis CJ, Rietz AC, Dobroff AS, Proneth B, Sidman RL, Pasqualini R, Arap W (2015) Selection and identification of ligand peptides targeting a model of castrate-resistant osteogenic prostate cancer and their receptors. Proc Natl Acad Sci USA 112(12): 3776-3781.

Mann AP, Scodeller P, Hussain S, Joo J, Kwon E, Braun GB, Molder T, She ZG, Kotamraju VR, Ranscht B, Krajewski S, Teesalu T, Bhatia S, Sailor MJ, Ruoslahti E (2016) A peptide for targeted, systemic delivery of imaging and therapeutic compounds into acute brain injuries. Nat Commun 7: 11980.

Matochko WL, Cory LiS, Tang SK, Derda R (2014) Prospective identification of parasitic sequences in phage display screens. Nucleic Acids Res 42(3): 1784-1798.

Robinson CW, Rye CS, Chessum NE, Jones K (2015) A model beta-sheet interaction and thermodynamic analysis of beta-strand mimetics. Org Biomol Chem 13(27): 7402-7407.

Shahidi N, Cheung WY (2016) Colorectal cancer screening: opportunities to improve uptake, outcomes, and disparities. World J Gastrointest Endosc 8(20): 733-740.

Siegel RL, Miller KD, Jemal A (2017) Cancer statistics, 2017. CA Cancer J Clin 67(1): 7-30.

Suzuki S, Sakata J, Utsumi F, Sekiya R, Kajiyama H, Shibata K, Kikkawa F, Nakatsura T (2016) Efficacy of glypican-3-derived peptide vaccine therapy on the survival of patients with refractory ovarian clear cell carcinoma. Oncoimmunology 5(11): e1238542.

Zhang Y, Chen J, Zhang Y, Hu Z, Hu D, Pan Y, Ou S, Liu G, Yin X, Zhao J, Ren L, Wang J (2007) Panning and identification of a colon tumor binding peptide from a phage display peptide library. J Biomol Screen 12(3): 429-435.

Zhu D, Qin Y, Wang J, Zhang L, Zou S, Zhu X, Zhu L (2016) Novel glypican3-binding peptide for in vivo hepatocellular carcinoma fluorescent imaging. Bioconjug Chem 27(3): 831-839.

This work is published under the standard license to publish agreement. After 12 months the work will become freely available and the license terms will switch to a Creative Commons AttributionNonCommercial-Share Alike 4.0 Unported License.

Supplementary Information accompanies this paper on British Journal of Cancer website (http://www.nature.com/bjc) 